\title{
Multidimensional Classical and Quantum Cosmology with Intersecting $p$-branes
}

\author{
V. D. Ivashchuk and V. N. Melnikov \\ Center for Gravitation and Fundamental Metrology \\ VNIIMS, 3-1 M. Ulyanovoy Str. \\ Moscow, 117313, Russia \\ e-mail: melnikov@fund.phys.msu.su
}

\begin{abstract}
Multidimensional cosmological model describing the evolution of $(n+1)$ Einstein spaces in the theory with several scalar fields and forms is considered. When a (electro-magnetic composite) $p$-brane Ansatz is adopted the field equations are reduced to the equations for Toda-like system. The Wheeler-De Witt equation is obtained. In the case when $n$ "internal" spaces are Ricci-flat, one space $M_{0}$ has a non-zero curvature, and all $p$-branes do not "live" in $M_{0}$, the classical and quantum solutions are obtained if certain orthogonality relations on parameters are imposed. Spherically-symmetric solutions with intersecting non-extremal $p$-branes are singled out. A non-orthogonal generalization of intersection rules corresponding to (open, closed) Toda lattices is obtained. A chain of bosonic $D \geq 11$ models (that may be related to hypothetical higher dimensional supergravities and $F$-theories) is suggested.
\end{abstract}

PACS number(s): 04.50.+h, 98.80.Hw, 04.60.Kz 


\section{Introduction}

In this paper we continue our investigations of $p$-brane solutions (see for example [2, 3, 13] and references therein) based on sigma-model approach [11, 16, 17] . (For pure gravitational sector see [18, 19].)

Here we consider a cosmological case, when all functions depend upon one variable (time). The model under consideration contains several scalar fields and antisymmetric forms and is governed by action (2.1).

The considered cosmological model contains some stringy cosmological models (see for example [10]. It may be obtained (at classical level) from multidimensional cosmological model with perfect fluid [24]-[28] as an interesting special case.

The paper is organized as follows. In Sect. 2 the model with composite electromagnetic p-branes is described. In Sect. 3 the $\sigma$-model representation (under certain constraints on $p$-branes) is considered and certain scalar invariants are calculated. In Sect. 4 the Wheeler-DeWitt equation is obtained. Sect. 5 is devoted to exact solutions (classical and quantum) for orthogonal case, when one factor space is curved. In Subsect. 5.3 spherically symmetric configurations with non-extremal $p$-branes is singled out. In

Sect. 6 the intersection rules are generalized to non-orthogonal case and a chain of bosonic $B_{D}$-models $(D \geq 11)$ containing "truncated" $D=11$ supergavity model [1] and $D=12$ model from 29] is suggested. (It may be supposed that these models or their analogues may be connected with higher dimensional generalization of $M$ - and $F$-theories [4, 65, 6, 7].)

\section{The model}

Here like in [16] we consider the model governed by the action

$$
\begin{aligned}
S=\quad & \frac{1}{2 \kappa^{2}} \int_{M} d^{D} z \sqrt{|g|}\left\{R[g]-2 \Lambda-h_{\alpha \beta} g^{M N} \partial_{M} \varphi^{\alpha} \partial_{N} \varphi^{\beta}\right. \\
& \left.-\sum_{a \in \Delta} \frac{\theta_{a}}{n_{a} !} \exp \left[2 \lambda_{a}(\varphi)\right]\left(F^{a}\right)_{g}^{2}\right\}+S_{G H},
\end{aligned}
$$

where $g=g_{M N} d z^{M} \otimes d z^{N}$ is the metric $(M, N=1, \ldots, D), \varphi=\left(\varphi^{\alpha}\right) \in \mathbb{R}^{l}$ is a vector from dilatonic scalar fields, $\left(h_{\alpha \beta}\right)$ is a non-degenerate $l \times l$ matrix $(l \in \mathbb{N}), \theta_{a}= \pm 1$,

$$
F^{a}=d A^{a}=\frac{1}{n_{a} !} F_{M_{1} \ldots M_{n_{a}}}^{a} d z^{M_{1}} \wedge \ldots \wedge d z^{M_{n_{a}}}
$$

is a $n_{a}$-form $\left(n_{a} \geq 1\right)$ on a $D$-dimensional manifold $M, \Lambda$ is cosmological constant and $\lambda_{a}$ is a 1 -form on $\mathbb{R}^{l}: \lambda_{a}(\varphi)=\lambda_{a \alpha} \varphi^{\alpha}, a \in \Delta, \alpha=1, \ldots, l$. In (2.1) we denote $|g|=\left|\operatorname{det}\left(g_{M N}\right)\right|$,

$$
\left(F^{a}\right)_{g}^{2}=F_{M_{1} \ldots M_{n_{a}}}^{a} F_{N_{1} \ldots N_{n_{a}}}^{a} g^{M_{1} N_{1}} \ldots g^{M_{n_{a}} N_{n_{a}}},
$$

$a \in \Delta$, where $\Delta$ is some finite set, and $S_{\mathrm{GH}}$ is the standard Gibbons-Hawking boundary term [23]. In the models with one time all $\theta_{a}=1$ when the signature of the metric is $(-1,+1, \ldots,+1)$. 
The equations of motion corresponding to (2.1) have the following form

$$
\begin{array}{r}
R_{M N}-\frac{1}{2} g_{M N} R=T_{M N}-\Lambda g_{M N}, \\
\triangle[g] \varphi^{\alpha}-\sum_{a \in \Delta} \theta_{a} \frac{\lambda_{a}^{\alpha}}{n_{a} !} e^{2 \lambda_{a}(\varphi)}\left(F^{a}\right)_{g}^{2}=0, \\
\nabla_{M_{1}}[g]\left(e^{2 \lambda_{a}(\varphi)} F^{a, M_{1} \ldots M_{n_{a}}}\right)=0,
\end{array}
$$

$a \in \Delta ; \alpha=1, \ldots, l$. In (2.5) $\lambda_{a}^{\alpha}=h^{\alpha \beta} \lambda_{a \beta}$, where $\left(h^{\alpha \beta}\right)$ is matrix inverse to $\left(h_{\alpha \beta}\right)$. In (2.4)

$$
T_{M N}=T_{M N}[\varphi, g]+\sum_{a \in \Delta} \theta_{a} e^{2 \lambda_{a}(\varphi)} T_{M N}\left[F^{a}, g\right]
$$

where

$$
\begin{array}{r}
T_{M N}[\varphi, g]=h_{\alpha \beta}\left(\partial_{M} \varphi^{\alpha} \partial_{N} \varphi^{\beta}-\frac{1}{2} g_{M N} \partial_{P} \varphi^{\alpha} \partial^{P} \varphi^{\beta}\right) \\
T_{M N}\left[F^{a}, g\right]=\frac{1}{n_{a} !}\left[-\frac{1}{2} g_{M N}\left(F^{a}\right)_{g}^{2}+n_{a} F_{M M_{2} \ldots M_{n_{a}}}^{a} F_{N}^{a, M_{2} \ldots M_{n_{a}}}\right] .
\end{array}
$$

In (2.5), (2.6) $\triangle[g]$ and $\nabla[g]$ are Laplace-Beltrami and covariant derivative operators respectively corresponding to $g$.

Let us consider the manifold

$$
M=\mathbb{R} \times M_{0} \times \ldots \times M_{n}
$$

with the metric

$$
g=w \mathrm{e}^{2 \gamma(u)} d u \otimes d u+\sum_{i=0}^{n} \mathrm{e}^{2 \phi^{i}(u)} g^{i},
$$

where $w= \pm 1, u$ is a distinguished coordinate which, by convention, will be called "time"; $g^{i}=g_{m_{i} n_{i}}^{i}\left(y_{i}\right) d y_{i}^{m_{i}} \otimes d y_{i}^{n_{i}}$ is a metric on $M_{i}$ satisfying the equation

$$
R_{m_{i} n_{i}}\left[g^{i}\right]=\xi_{i} g_{m_{i} n_{i}}^{i}
$$

$m_{i}, n_{i}=1, \ldots, d_{i} ; d_{i}=\operatorname{dim} M_{i}, \xi_{i}=$ const, $i=0, \ldots, n ; n \in \mathbf{N}$. Thus, $\left(M_{i}, g^{i}\right)$ are Einstein spaces. The functions $\gamma, \phi^{i}:\left(u_{-}, u_{+}\right) \rightarrow \mathbf{R}$ are smooth.

Each manifold $M_{i}$ is assumed to be oriented and connected, $i=0, \ldots, n$. Then the volume $d_{i}$-form

$$
\tau_{i}=\sqrt{\left|g^{i}\left(y_{i}\right)\right|} d y_{i}^{1} \wedge \ldots \wedge d y_{i}^{d_{i}}
$$

and the signature parameter

$$
\varepsilon(i)=\operatorname{sign} \operatorname{det}\left(g_{m_{i} n_{i}}^{i}\right)= \pm 1
$$

are correctly defined for all $i=0, \ldots, n$.

Let

$$
\Omega_{0}=\{\emptyset,\{0\},\{1\}, \ldots,\{n\},\{0,1\}, \ldots,\{0,1, \ldots, n\}\}
$$

be a set of all subsets of

$$
I_{0} \equiv\{0, \ldots, n\}
$$


For any $I=\left\{i_{1}, \ldots, i_{k}\right\} \in \Omega_{0}, i_{1}<\ldots<i_{k}$, we define a form

$$
\tau(I) \equiv \tau_{i_{1}} \wedge \ldots \wedge \tau_{i_{k}},
$$

of rank

$$
d(I) \equiv \sum_{i \in I} d_{i}=d_{i_{1}}+\ldots+d_{i_{k}},
$$

and a corresponding $p$-brane submanifold

$$
M_{I} \equiv M_{i_{1}} \times \ldots \times M_{i_{k}},
$$

where $p=d(I)-1\left(\operatorname{dimM}_{\mathrm{I}}=d(I)\right)$. We also define $\varepsilon$-symbol

$$
\varepsilon(I) \equiv \varepsilon\left(i_{1}\right) \ldots \varepsilon\left(i_{k}\right) .
$$

For $I=\emptyset$ we put $\tau(\emptyset)=\varepsilon(\emptyset)=1, d(\emptyset)=0$.

For fields of forms we adopt the following "composite electro-magnetic" Ansatz

$$
F^{a}=\sum_{I \in \Omega_{a, e}} F^{(a, e, I)}+\sum_{J \in \Omega_{a, m}} F^{(a, m, J)},
$$

where

$$
\begin{aligned}
F^{(a, e, I)}=d \Phi^{(a, e, I)} & \wedge \tau(I), \\
F^{(a, m, J)}=\mathrm{e}^{-2 \lambda_{a}(\varphi)} *\left(d \Phi^{(a, m, J)}\right. & \wedge \tau(J)),
\end{aligned}
$$

$a \in \Delta, I \in \Omega_{a, e}, J \in \Omega_{a, m}$ and

$$
\Omega_{a, e}, \Omega_{a, m} \subset \Omega_{0} .
$$

(For empty $\Omega_{a, v}=\emptyset, v=e, m$, we put $\sum_{\emptyset}=0$ in (2.21)). In $(2.23) *=*[g]$ is the Hodge operator on $(M, g)$.

For the potentials in (2.22), (2.23) we put

$$
\Phi^{s}=\Phi^{s}(u)
$$

$s \in S$, where

$$
S=S_{e} \sqcup S_{m}, \quad S_{v} \equiv \coprod_{a \in \Delta}\{a\} \times\{v\} \times \Omega_{a, v},
$$

$v=e, m$.

For dilatonic scalar fields we put

$$
\varphi^{\alpha}=\varphi^{\alpha}(u),
$$

$\alpha=1, \ldots, l$.

¿From (2.22) and (2.23) we obtain the relations between dimensions of $p$-brane worldsheets and ranks of forms

$$
\begin{array}{r}
d(I)=n_{a}-1, \quad I \in \Omega_{a, e}, \\
d(J)=D-n_{a}-1, \quad J \in \Omega_{a, m},
\end{array}
$$

in electric and magnetic cases respectively. 


\section{$3 \quad \sigma$-model representation}

Restrictions on $\Omega_{a, v}$. Let

$$
w_{1} \equiv\left\{i \mid i \in\{1, \ldots, n\}, d_{i}=1\right\} .
$$

The set $w_{1}$ describes all 1-dimensional manifolds among $M_{i}(i \geq 0)$. We impose the following restrictions on the sets $\Omega_{a, v}(2.24)$ :

$$
W_{i j}\left(\Omega_{a, v}\right)=\emptyset,
$$

$a \in \Delta ; v=e, m ; i, j \in w_{1}, i<j$ and

$$
W_{j}^{(1)}\left(\Omega_{a, m}, \Omega_{a, e}\right)=\emptyset,
$$

$a \in \Delta ; j \in w_{1}$. Here

$$
W_{i j}\left(\Omega_{*}\right) \equiv\left\{(I, J) \mid I, J \in \Omega_{*}, I=\{i\} \sqcup(I \cap J), J=\{j\} \sqcup(I \cap J)\right\},
$$

$i, j \in w_{1}, i \neq j, \Omega_{*} \subset \Omega_{0}$ and

$$
W_{j}^{(1)}\left(\Omega_{a, m}, \Omega_{a, e}\right) \equiv\left\{(I, J) \in \Omega_{a, m} \times \Omega_{a, e} \mid \bar{I}=\{j\} \sqcup J\right\},
$$

$j \in w_{1}$. In (3.5)

$$
\bar{I} \equiv I_{0} \backslash I
$$

is "dual" set. (The restrictions (3.2) and (3.3) are trivially satisfied when $n_{1} \leq 1$ and $n_{1}=0$ respectively, where $n_{1}=\left|w_{1}\right|$ is the number of 1-dimensional manifolds among $\left.M_{i}\right)$.

It follows from [16] (see Proposition 2 in [16]) that the equations of motion (2.4)-(2.6) and the Bianchi identities

$$
d F^{s}=0, \quad s \in S
$$

for the field configuration (2.11), (2.21)-(2.25), (2.27) with the restrictions (3.2), (3.3) imposed are equivalent to equations of motion for $\sigma$-model with the action

$$
S_{\sigma}=\frac{\mu}{2} \int d u \mathcal{N}\left\{G_{i j} \dot{\phi}^{i} \dot{\phi}^{j}+h_{\alpha \beta} \dot{\varphi}^{\alpha} \dot{\varphi}^{\beta}+\sum_{s \in S} \varepsilon_{s} \exp \left[-2 U^{s}(\phi, \varphi)\right]\left(\dot{\Phi}^{s}\right)^{2}-2 \mathcal{N}^{-2} V(\phi)\right\},
$$

where $\dot{x} \equiv d x / d u$,

$$
V=V(\phi)=-w \Lambda \mathrm{e}^{2 \gamma_{0}(\phi)}+\frac{w}{2} \sum_{i=0}^{n} \xi_{i} d_{i} \mathrm{e}^{-2 \phi^{i}+2 \gamma_{0}(\phi)}
$$

is the potential with

$$
\gamma_{0}(\phi) \equiv \sum_{i=0}^{n} d_{i} \phi^{i}
$$

and

$$
\mathcal{N}=\exp \left(\gamma_{0}-\gamma\right)>0
$$


is the lapse function,

$$
\begin{aligned}
U^{s}=U^{s}(\phi, \varphi) & =-\chi_{s} \lambda_{a_{s}}(\varphi)+\sum_{i \in I_{s}} d_{i} \phi^{i}, \\
\varepsilon_{s} & =(-\varepsilon[g])^{\left(1-\chi_{s}\right) / 2} \varepsilon\left(I_{s}\right) \theta_{a_{s}}
\end{aligned}
$$

for $s=\left(a_{s}, v_{s}, I_{s}\right) \in S, \varepsilon[g]=\operatorname{sign} \operatorname{det}\left(g_{M N}\right)$,

$$
\begin{gathered}
\chi_{s}=+1, \quad v_{s}=e ; \\
\chi_{s}=-1, \quad v_{s}=m,
\end{gathered}
$$

and

$$
G_{i j}=d_{i} \delta_{i j}-d_{i} d_{j}
$$

are components of the "pure cosmological" minisupermetric, $i, j=0, \ldots, n[22]$.

In the electric case $\left(F^{(a, m, I)}=0\right)$ for finite internal space volumes $V_{i}$ the action (3.8) coincides with the action (2.1) if $\mu=-w / \kappa_{0}^{2}, \kappa^{2}=\kappa_{0}^{2} V_{0} \ldots V_{n}$.

Action (3.8) may be also written in the form

$$
S_{\sigma}=\frac{\mu}{2} \int d u \mathcal{N}\left\{\mathcal{G}_{\hat{A} \hat{B}}(X) \dot{X}^{\hat{A}} \dot{X}^{\hat{B}}-2 \mathcal{N}^{-2} V(X)\right\}
$$

where $X=\left(X^{\hat{A}}\right)=\left(\phi^{i}, \varphi^{\alpha}, \Phi^{s}\right) \in \mathbf{R}^{N}$, and minisupermetric

$$
\mathcal{G}=\mathcal{G}_{\hat{A} \hat{B}}(X) d X^{\hat{A}} \otimes d X^{\hat{B}}
$$

on minisuperspace

$$
\mathcal{M}=\mathbf{R}^{N}, \quad N=n+1+l+|S|
$$

$(|S|$ is the number of elements in $S)$ is defined by the relation

$$
\left(\mathcal{G}_{\hat{A} \hat{B}}(X)\right)=\left(\begin{array}{ccc}
G_{i j} & 0 & 0 \\
0 & h_{\alpha \beta} & 0 \\
0 & 0 & \varepsilon_{s} \mathrm{e}^{-2 U^{s}(X)} \delta_{s s^{\prime}}
\end{array}\right) .
$$

\subsection{Scalar products}

The minisuperspace metric (3.18) may be also written in the form

$$
\mathcal{G}=\bar{G}+\sum_{s \in S} \varepsilon_{s} \mathrm{e}^{-2 U^{s}(x)} d \Phi^{s} \otimes d \Phi^{s}
$$

where $x=\left(x^{A}\right)=\left(\phi^{i}, \varphi^{\alpha}\right)$,

$$
\begin{array}{r}
\bar{G}=\bar{G}_{A B} d x^{A} \otimes d x^{B}=G_{i j} d \phi^{i} \otimes d \phi^{j}+h_{\alpha \beta} d \varphi^{\alpha} \otimes d \varphi^{\beta}, \\
\left(\bar{G}_{A B}\right)=\left(\begin{array}{cc}
G_{i j} & 0 \\
0 & h_{\alpha \beta}
\end{array}\right),
\end{array}
$$


$U^{s}(x)=U_{A}^{s} x^{A}$ is defined in (3.12) and

$$
\left(U_{A}^{s}\right)=\left(d_{i} \delta_{i I_{s}},-\chi_{s} \lambda_{a_{s} \alpha}\right)
$$

Here

$$
\delta_{i I} \equiv \sum_{j \in I} \delta_{i j}=\begin{array}{ll}
1, & i \in I \\
0, & i \notin I
\end{array}
$$

is an indicator of $i$ belonging to $I$. The potential (3.9) reads

$$
V=(-w \Lambda) \mathrm{e}^{2 U^{\Lambda}(x)}+\sum_{j=0}^{n} \frac{w}{2} \xi_{j} d_{j} \mathrm{e}^{2 U^{j}(x)},
$$

where

$$
\begin{array}{r}
U^{j}(x)=U_{A}^{j} x^{A}=-\phi^{j}+\gamma_{0}(\phi), \\
U^{\Lambda}(x)=U_{A}^{\Lambda} x^{A}=\gamma_{0}(\phi), \\
\left(U_{A}^{j}\right)=\left(-\delta_{i}^{j}+d_{i}, 0\right), \\
\left(U_{A}^{\Lambda}\right)=\left(d_{i}, 0\right) .
\end{array}
$$

The integrability of the Lagrange system (3.17) crucially depends upon the scalar products of co-vectors $U^{\Lambda}, U^{j}, U^{s}$ corresponding to $\bar{G}$ :

$$
\left(U, U^{\prime}\right)=\bar{G}^{A B} U_{A} U_{B}^{\prime},
$$

where

$$
\left(\bar{G}^{A B}\right)=\left(\begin{array}{cc}
G^{i j} & 0 \\
0 & h^{\alpha \beta}
\end{array}\right)
$$

is matrix inverse to (3.23). Here (as in 22])

$$
G^{i j}=\frac{\delta^{i j}}{d_{i}}+\frac{1}{2-D}
$$

$i, j=0, \ldots, n$. These products have the following form

$$
\begin{array}{r}
\left(U^{i}, U^{j}\right)=\frac{\delta_{i j}}{d_{j}}-1, \\
\left(U^{i}, U^{\Lambda}\right)=-1, \\
\left(U^{\Lambda}, U^{\Lambda}\right)=-\frac{D-1}{D-2}, \\
\left(U^{s}, U^{s^{\prime}}\right)=q\left(I_{s}, I_{s^{\prime}}\right)+\chi_{s} \chi_{s^{\prime}} \lambda_{a_{s}} \cdot \lambda_{a_{s^{\prime}}}, \\
\left(U^{s}, U^{i}\right)=-\delta_{i I_{s}}, \\
\left(U^{s}, U^{\Lambda}\right)=\frac{d\left(I_{s}\right)}{2-D},
\end{array}
$$

where $s=\left(a_{s}, v_{s}, I_{s}\right), s^{\prime}=\left(a_{s^{\prime}}, v_{s^{\prime}}, I_{s^{\prime}}\right) \in S$,

$$
\begin{array}{r}
q(I, J) \equiv d(I \cap J)+\frac{d(I) d(J)}{2-D}, \\
\lambda_{a} \cdot \lambda_{b} \equiv \lambda_{a \alpha} \lambda_{b \beta} h^{\alpha \beta} .
\end{array}
$$

Relations (3.34)-(3.36) were found in 28 and (3.37) in 16]. 


\section{Wheeler-De Witt equation}

Here we fix the gauge as follows

$$
\gamma_{0}-\gamma=f(X), \quad \mathcal{N}=e^{f},
$$

where $f: \mathcal{M} \rightarrow \mathbf{R}$ is a smooth function. Then we obtain the Lagrange system with the Lagrangian

$$
L_{f}=\frac{\mu}{2} \mathrm{e}^{f} \mathcal{G}_{\hat{A} \hat{B}}(X) \dot{X}^{\hat{A}} \dot{X}^{\hat{B}}-\mu \mathrm{e}^{-f} V
$$

and the energy constraint

$$
E_{f}=\frac{\mu}{2} \mathrm{e}^{f} \mathcal{G}_{\hat{A} \hat{B}}(X) \dot{X}^{\hat{A}} \dot{X}^{\hat{B}}+\mu \mathrm{e}^{-f} V=0 .
$$

Using the standard prescriptions of (covariant and conformally covarint) quantization (see, for example, [20, 21, 22]) we are led to the Wheeler-DeWitt (WDW) equation

$$
\hat{H}^{f} \Psi^{f} \equiv\left(-\frac{1}{2 \mu} \Delta\left[e^{f} \mathcal{G}\right]+\frac{a}{\mu} R\left[e^{f} \mathcal{G}\right]+e^{-f} \mu V\right) \Psi^{f}=0,
$$

where

$$
a=a_{N}=\frac{N-2}{8(N-1)} .
$$

Here $\Psi^{f}=\Psi^{f}(X)$ is the so-called "wave function of the universe" corresponding to the $f$-gauge (4.1), and satisfying the relation

$$
\Psi^{f}=e^{b f} \Psi^{f=0}, \quad b=(2-N) / 2,
$$

$\left(\Delta\left[\mathcal{G}_{1}\right]\right.$ and $R\left[\mathcal{G}_{1}\right]$ denote the Laplace-Beltrami operator and the scalar curvature corresponding to $\mathcal{G}_{1}$ ).

For the scalar curvature of minisupermetric (3.21) we get (see (2.29) in [16])

$$
R[\mathcal{G}]=-\sum_{s \in S}\left(U^{s}, U^{s}\right)-\sum_{s, s^{\prime} \in S}\left(U^{s}, U^{s^{\prime}}\right) .
$$

For the Laplace operator we obtain

$$
\Delta[\mathcal{G}]=\mathrm{e}^{U(x)} \frac{\partial}{\partial x^{A}}\left(\bar{G}^{A B} \mathrm{e}^{-U(x)} \frac{\partial}{\partial x^{B}}\right)+\sum_{s \in S} \varepsilon_{s} \mathrm{e}^{2 U^{s}(x)}\left(\frac{\partial}{\partial \Phi^{s}}\right)^{2},
$$

where $U(x)=\sum_{s \in S} U^{s}(x)$.

Harmonic-time gauge. The WDW equation (4.4) for $f=0$

$$
\hat{H} \Psi \equiv\left(-\frac{1}{2 \mu} \Delta[\mathcal{G}]+\frac{a}{\mu} R[\mathcal{G}]+\mu V\right) \Psi=0,
$$

may be rewritten, using relations (4.7), (4.8) and

$$
U^{s i}=G^{i j} U_{j}^{s}=\delta_{i I_{s}}-\frac{d\left(I_{s}\right)}{D-2}, \quad U^{s \alpha}=-\chi_{s} \lambda_{a_{s}}^{\alpha},
$$


as follows

$$
\begin{gathered}
2 \mu \hat{H} \Psi=\left\{-G^{i j} \frac{\partial}{\partial \phi^{i}} \frac{\partial}{\partial \phi^{j}}-h^{\alpha \beta} \frac{\partial}{\partial \varphi^{\alpha}} \frac{\partial}{\partial \varphi^{\beta}}-\sum_{s \in S} \varepsilon_{s} \mathrm{e}^{2 U^{s}(\phi, \varphi)}\left(\frac{\partial}{\partial \Phi^{s}}\right)^{2}\right. \\
\left.+\sum_{s \in S}\left[\sum_{i \in I_{s}} \frac{\partial}{\partial \phi^{i}}-\frac{d\left(I_{s}\right)}{D-2} \sum_{j=0}^{n} \frac{\partial}{\partial \phi^{j}}-\chi_{s} \lambda_{a_{s}}^{\alpha} \frac{\partial}{\partial \varphi^{\alpha}}\right]+2 a R[\mathcal{G}]+2 \mu^{2} V\right\} \Psi=0 .
\end{gathered}
$$

Here $\hat{H} \equiv \hat{H}^{f=0}$ and $\Psi \equiv \Psi^{f=0}$.

\section{Exact solutions with one curved factor space and orthogonal $U^{s}$}

Here we put the following restrictions on the parameters of the model

$$
\text { (i) } \quad \Lambda=0 \text {, }
$$

i.e. the cosmological constant is zero,

$$
\text { (ii) } \quad \xi_{0} \neq 0, \quad \xi_{1}=\ldots=\xi_{n}=0,
$$

i.e. one space is curved and others are Ricci-flat,

$$
\text { (iii) } 0 \notin I_{s}, \quad \forall s=\left(a_{s}, v_{s}, I_{s}\right) \in S \text {, }
$$

i.e. all "brane" submanifolds $M_{I_{s}}$ (see (2.19)) do not contain $M_{0}$.

We also put the following orthogonality restriction on the vectors $U^{s}$

$$
\left(\text { iv) }\left(U^{s}, U^{s^{\prime}}\right)=d\left(I_{s} \cap I_{s^{\prime}}\right)+\frac{d\left(I_{s}\right) d\left(I_{s^{\prime}}\right)}{2-D}+\chi_{s} \chi_{s^{\prime}} \lambda_{a_{s} \alpha} \lambda_{a_{s^{\prime}} \beta} h^{\alpha \beta}=0\right. \text {, }
$$

for $s \neq s^{\prime}, s=\left(a_{s}, v_{s}, I_{s}\right), s^{\prime}=\left(a_{s^{\prime}}, v_{s^{\prime}}, I_{s^{\prime}}\right) \in S$ and

$$
\text { (v) } \quad\left(U^{s}, U^{s}\right) \neq 0
$$

for all $s \in S$.

¿From (i), (ii) we get for the potential (3.26)

$$
V=\frac{1}{2} w \xi_{0} d_{0} \mathrm{e}^{2 U^{0}(x)},
$$

where

$$
\left(U^{0}, U^{0}\right)=\frac{1}{d_{0}}-1<0
$$

(see (3.34)).

¿From (iii) and (3.38) we get

$$
\left(U^{0}, U^{s}\right)=0
$$


for all $s \in S$. Thus, all co-vectors $U^{0}, U^{s}(s \in S)$ belonging to dual space $\left(\mathbf{R}^{n+1+l}\right)^{*} \simeq$ $\mathbf{R}^{n+1+l}$ are orthogonal with respect to the scalar product (3.31).

Let

$$
\eta_{s} \equiv \operatorname{sign}\left(U^{s}, U^{s}\right)
$$

and $|S|_{ \pm}$is the number of $U^{s}$ with $\eta_{s}= \pm 1 ;|S|_{+}+|S|_{-}=|S|$. Let the matrix $\left(h_{\alpha \beta}\right)$ has the signature $(\underbrace{-1, \ldots,-1}_{l_{-}}, \underbrace{+1, \ldots,+1}_{l_{+}})\left(l_{-}+l_{+}=l\right)$. The matrix $\left(G_{i j}\right)$ has a pseudoEuclidean signature $(-1, \underbrace{+1, \ldots,+1}_{n}$ [22]. Then from $(5.7)-(5.8)$ we obtain $|S|_{-} \leq l_{-}$, $|S|_{+} \leq n+l_{+}$and hence

$$
|S| \leq n+l \text {. }
$$

Thus we obtain the restriction on the number of orthogonal (in minisupermetric (3.32) p-brane configurations.

\subsection{Quantum solutions}

The truncated minisuperspace metric (3.23) may be diagonalized by the linear transformation

$$
z^{A}=S_{B}^{A} x^{B}, \quad\left(z^{A}\right)=\left(z^{0}, z^{a}, z^{s}\right)
$$

as follows

$$
\bar{G}=\bar{G}_{A B} d x^{A} \otimes d x^{B}=-d z^{0} \otimes d z^{0}+\sum_{s \in S} \eta_{s} d z^{s} \otimes d z^{s}+d z^{a} \otimes d z^{b} \eta_{a b}
$$

where $a, b=1, \ldots, n+l-|S| ; \eta_{a b}=\eta_{a a} \delta_{a b} ; \eta_{a a}= \pm 1$, and

$$
\begin{gathered}
q_{0} z^{0}=U^{0}(x), \\
q_{s} z^{s}=U^{s}(x),
\end{gathered}
$$

with

$$
\begin{array}{r}
q_{0} \equiv \sqrt{\left|\left(U^{0}, U^{0}\right)\right|}=\sqrt{1-\frac{1}{d_{0}}}>0, \\
q_{s}=\nu_{s}^{-1} \equiv \sqrt{\left|\left(U^{s}, U^{s}\right)\right|}=\sqrt{\left|d\left(I_{s}\right)\left(1+\frac{d\left(I_{s}\right)}{2-D}\right)+\lambda_{a_{s}}^{2}\right|}>0,
\end{array}
$$

$s=\left(a_{s}, v_{s}, I_{s}\right) \in S$.

¿From (4.8), (5.11), (5.12) and (5.14) we get

$$
\begin{array}{r}
\Delta[\mathcal{G}]=-\left(\frac{\partial}{\partial z^{0}}\right)^{2}+\eta^{a b} \frac{\partial}{\partial z^{a}} \frac{\partial}{\partial z^{b}}+\sum_{s \in S} \eta_{s} \mathrm{e}^{q_{s} z^{s}} \frac{\partial}{\partial z^{s}}\left(\mathrm{e}^{-q_{s} z^{s}} \frac{\partial}{\partial z^{s}}\right) \\
+\sum_{s \in S} \varepsilon_{s} \mathrm{e}^{2 q_{s} z^{s}}\left(\frac{\partial}{\partial \Phi^{s}}\right)^{2} .
\end{array}
$$


The relation (4.7) in the orthogonal case reads as

$$
R[\mathcal{G}]=-2 \sum_{s \in S}\left(U^{s}, U^{s}\right)=-2 \sum_{s \in S} \eta_{s} q_{s}^{2}
$$

We are seeking the solution to WDW equation (4.9) by the method of the separation of variables, i.e. we put

$$
\Psi_{*}(z)=\Psi_{0}\left(z^{0}\right)\left(\prod_{s \in S} \Psi_{s}\left(z^{s}\right)\right) \mathrm{e}^{\mathrm{i} P_{s} \Phi^{s}} \mathrm{e}^{\mathrm{i} p_{a} z^{a}} .
$$

It follows from (5.17) that $\Psi_{*}(z)$ satisfies WDW equation (4.9) if

$$
\begin{array}{r}
2 \hat{H}_{0} \Psi_{0} \equiv\left\{\left(\frac{\partial}{\partial z^{0}}\right)^{2}+\mu^{2} w \xi_{0} d_{0} \mathrm{e}^{2 q_{0} z^{0}}\right\} \Psi_{0}=2 \mathcal{E}_{0} \Psi_{0} \\
2 \hat{H}_{s} \Psi_{s} \equiv\left\{-\eta_{s} \mathrm{e}^{q_{s} z^{s}} \frac{\partial}{\partial z^{s}}\left(\mathrm{e}^{-q_{s} z^{s}} \frac{\partial}{\partial z^{s}}\right)+\varepsilon_{s} P_{s}^{2} \mathrm{e}^{2 q_{s} z^{s}}\right\} \Psi_{s}=2 \mathcal{E}_{s} \Psi_{s},
\end{array}
$$

$s \in S$, and

$$
2 \mathcal{E}_{0}+\eta^{a b} p_{a} p_{b}+2 \sum_{s \in S} \mathcal{E}_{s}+2 a R[\mathcal{G}]=0
$$

with $a$ and $R[\mathcal{G}]$ from $(4.5)$ and (5.18) respectively.

Using the relations from Appendix 1 we obtain linearly independent solutions to (5.20) and (5.21) respectively

$$
\begin{gathered}
\Psi_{0}\left(z^{0}\right)=B_{\omega_{0}\left(\mathcal{E}_{0}\right)}^{0}\left(\sqrt{-w \mu^{2} \xi_{0} d_{0}} \frac{\mathrm{e}^{q_{0} z^{0}}}{q_{0}}\right) \\
\Psi_{s}\left(z^{s}\right)=\mathrm{e}^{q_{s} z^{s} / 2} B_{\omega_{s}\left(\mathcal{E}_{s}\right)}^{s}\left(\sqrt{\eta_{s} \varepsilon_{s} P_{s}^{2}} \frac{\mathrm{e}^{q_{s} z^{s}}}{q_{s}}\right)
\end{gathered}
$$

where

$$
\omega_{0}\left(\mathcal{E}_{0}\right)=\sqrt{2 \mathcal{E}_{0}} / q_{0}, \quad \omega_{s}\left(\mathcal{E}_{s}\right)=\sqrt{\frac{1}{4}-2 \eta_{s} \mathcal{E}_{s} \nu_{s}^{2}},
$$

$s \in S$ and $B_{\omega}^{0}, B_{\omega}^{s}=I_{\omega}, K_{\omega}$ are the modified Bessel function.

The general solution of the WDW equation (4.9) is a superposition of the "separated" solutions (5.19):

$$
\Psi(z)=\sum_{B} \int d p d P d \mathcal{E} C(p, P, \mathcal{E}, B) \Psi_{*}(z \mid p, P, \mathcal{E}, B)
$$

where $p=\left(p_{a}\right), P=\left(P_{s}\right), \mathcal{E}=\left(\mathcal{E}_{s}\right), B=\left(B^{0}, B^{s}\right), B^{0}, B^{s}=I, K ;$ and $\Psi_{*}=$ $\Psi_{*}(z \mid p, P, \mathcal{E}, B)$ is given by relation (5.19), (5.23)-(5.25) with $\mathcal{E}_{0}$ from (5.22). Here $C(p, P, \mathcal{E}, B)$ are smooth enough functions. In non-composite electric case these solutions were considered recently in [35]. 


\subsection{Classical solutions}

\subsubsection{Toda-like representation.}

Here we will integrate the Lagrange equations corresponding to the Lagrangian (4.2) with the energy-constraint (4.3) and hence we will find classical exact solutions when the restrictions (5.1)-(5.5) are imposed. We put $f=0$, i.e. the harmonic time gauge is considered.

The problem of integrability may be simplified if we integrate the Maxwell equations (for $s \in S_{e}$ ) and Bianchi identities (for $s \in S_{m}$ ):

$$
\frac{d}{d u}\left(\exp \left(-2 U^{s}\right) \dot{\Phi}^{s}\right)=0 \Longleftrightarrow \dot{\Phi}^{s}=Q_{s} \exp \left(2 U^{s}\right)
$$

where $Q_{s}$ are constants, $s=\left(a_{s}, v_{s}, I_{s}\right) \in S$.

Let

$$
\begin{aligned}
& Q_{s} \neq 0, \quad s \in S_{*} ; \\
& Q_{s}=0, \quad s \in S \backslash S_{*},
\end{aligned}
$$

where $S_{*} \subset S$ is a non-empty subset of $S$.

For fixed $Q=\left(Q_{s}, s \in S_{*}\right)$ the Lagrange equations for the Lagrangian (4.2) with $f=0$ corresponding to $\left(x^{A}\right)=\left(\phi^{i}, \varphi^{\alpha}\right)$, when equations (5.27) are substituted are equivalent to the Lagrange equations for the Lagrangian

$$
L_{Q}=\frac{1}{2} \bar{G}_{A B} \dot{x}^{A} \dot{x}^{B}-V_{Q}
$$

where

$$
V_{Q}=V+\frac{1}{2} \sum_{s \in S_{*}} \varepsilon_{s} Q_{s}^{2} \exp \left[2 U^{s}(x)\right]
$$

$\left(\bar{G}_{A B}\right)$ and $V$ are defined in $(3.23)$ and (3.9) respectively. The zero-energy constraint (4.3) reads

$$
E_{Q}=\frac{1}{2} \bar{G}_{A B} \dot{x}^{A} \dot{x}^{B}+V_{Q}=0 .
$$

\subsubsection{Exact solutions for one curved space and orthogonal $U^{s}$.}

When the conditions (i) $-(\mathbf{v})$ are satisfied exact solutions for Lagrangian (5.29) with the potential (5.30) and $V$ from (5.6) could readily obtained using the relations from Appendix 2.

The solutions read:

$$
x^{A}(u)=-\frac{U^{0 A}}{\left(U^{0}, U^{0}\right)} \ln \left|f_{0}\left(u-u_{0}\right)\right|-\sum_{s \in S_{*}} \frac{U^{s A}}{\left(U^{s}, U^{s}\right)} \ln \left|f_{s}\left(u-u_{s}\right)\right|+c^{A} u+\bar{c}^{A},
$$

where $u_{0}, u_{s}$ are constants, $s \in S_{*}$. Functions $f_{0}$ and $f_{s}$ in (5.32) are the following:

$$
f_{0}(\tau)=\left|\frac{\xi_{0}\left(d_{0}-1\right)}{C_{0}}\right|^{1 / 2} \operatorname{sh}\left(\sqrt{C_{0}} \tau\right), C_{0}>0, \xi_{0} w>0
$$




$$
\begin{array}{r}
\left|\frac{\xi_{0}\left(d_{0}-1\right)}{C_{0}}\right|^{1 / 2} \sin \left(\sqrt{\left|C_{0}\right|} \tau\right), C_{0}<0, \xi_{0} w>0 \\
\left|\frac{\xi_{0}\left(d_{0}-1\right)}{C_{0}}\right|^{1 / 2} \operatorname{ch}\left(\sqrt{C_{0}} \tau\right), C_{0}>0, \xi_{0} w<0 \\
\left|\xi_{0}\left(d_{0}-1\right)\right|^{1 / 2} \tau, C_{0}=0, \xi_{0} w>0
\end{array}
$$

and

$$
\begin{array}{r}
f_{s}(\tau)=\frac{\left|Q_{s}\right|}{\nu_{s}\left|C_{s}\right|^{1 / 2}} \operatorname{sh}\left(\sqrt{C_{s}} \tau\right), C_{s}>0, \eta_{s} \varepsilon_{s}<0 ; \\
\frac{\left|Q_{s}\right|}{\nu_{s}\left|C_{s}\right|^{1 / 2}} \sin \left(\sqrt{\left|C_{s}\right|} \tau\right), C_{s}<0, \eta_{s} \varepsilon_{s}<0 ; \\
\frac{\left|Q_{s}\right|}{\nu_{s}\left|C_{s}\right|^{1 / 2}} \operatorname{ch}\left(\sqrt{C_{s}} \tau\right), C_{s}>0, \eta_{s} \varepsilon_{s}>0 ; \\
\frac{\left|Q^{s}\right|}{\nu_{s}} \tau, C_{s}=0, \eta_{s} \varepsilon_{s}<0,
\end{array}
$$

where $C_{0}$ and $C_{s}$ are constants. Here we used the relations (5.7)-(5.9). The contravariant components $U^{0 A}=\bar{G}^{A B} U_{B}^{0}$ are

$$
U^{0 i}=-\frac{\delta_{0}^{i}}{d_{0}}, \quad U^{0 \alpha}=0 .
$$

Corresponding relations for $U^{s A}, s \in S$, were presented in (4.10).

Using (5.32), (5.7), (5.16), 4.10) and (5.41) we obtain

$$
\phi^{i}=\frac{\delta_{0}^{i}}{1-d_{0}} \ln \left|f_{0}\right|+\sum_{s \in S_{*}} \alpha_{s}^{i} \ln \left|f_{s}\right|+c^{i} u+\bar{c}^{i},
$$

where

$$
\alpha_{s}^{i}=-\eta_{s} \nu_{s}^{2}\left(\delta_{i I_{s}}-\frac{d\left(I_{s}\right)}{D-2}\right)
$$

$s \in S$, and

$$
\varphi^{\alpha}=\sum_{s \in S_{*}} \eta_{s} \nu_{s}^{2} \chi_{s} \lambda_{a_{s}}^{\alpha} \ln \left|f_{s}\right|+c^{\alpha} u+\bar{c}^{\alpha}
$$

$\alpha=1, \ldots, l$.

Vectors $c=\left(c^{A}\right)$ and $\bar{c}=\left(\bar{c}^{A}\right)$ satisfy the linear constraint relations (see Appendix 2)

$$
\begin{array}{r}
U^{0}(c)=U_{A}^{0} c^{A}=-c^{0}+\sum_{j=0}^{n} d_{j} c^{j}=0, \\
U^{0}(\bar{c})=U_{A}^{0} \bar{c}^{A}=-\bar{c}^{0}+\sum_{j=0}^{n} d_{j} \bar{c}^{j}=0, \\
U^{s}(c)=U_{A}^{s} c^{A}=\sum_{i \in I_{s}} d_{i} c^{i}-\chi_{s} \lambda_{a_{s} \alpha} c^{\alpha}=0, \\
U^{s}(\bar{c})=U_{A}^{s} \bar{c}^{A}=\sum_{i \in I_{s}} d_{i} \bar{c}^{i}-\chi_{s} \lambda_{a_{s} \alpha} \bar{c}^{\alpha}=0,
\end{array}
$$


$s \in S$. The harmonic gauge function (3.10) reads

$$
\gamma_{0}(\phi)=\frac{d_{0}}{1-d_{0}} \ln \left|f_{0}\right|+\sum_{s \in S_{*}} \frac{d\left(I_{s}\right)}{D-2} \eta_{s} \nu_{s}^{2} \ln \left|f_{s}\right|+c^{0} u+\bar{c}^{0} .
$$

The zero-energy constraint reads (see Appendix 2)

$$
E=E_{0}+\sum_{s \in S_{*}} E_{s}+\frac{1}{2} \bar{G}_{A B} c^{A} c^{B}=0
$$

where $C_{0}=2 E_{0}\left(U^{0}, U^{0}\right), C_{s}=2 E_{s}\left(U^{s}, U^{s}\right)$. Using the relations (5.15), (5.16), (5.45) we rewrite $(5.50)$ as

$$
C_{0} \frac{d_{0}}{d_{0}-1}=\sum_{s \in S_{*}} C_{s} \nu_{s}^{2} \eta_{s}+h_{\alpha \beta} c^{\alpha} c^{\beta}+\sum_{i=1}^{n} d_{i}\left(c^{i}\right)^{2}+\frac{1}{d_{0}-1}\left(\sum_{i=1}^{n} d_{i} c^{i}\right)^{2} .
$$

¿From relation

$$
\exp 2 U^{s}=f_{s}^{-2}
$$

following from (5.32), (5.4), (5.8), (5.47), (5.48) we get for electric-type forms (2.22)

$$
F^{s}=Q_{s} f_{s}^{-2} d u \wedge \tau\left(I_{s}\right),
$$

$s \in S_{e}$, and for magnetic-type forms (2.23)

$$
F^{s}=\mathrm{e}^{-2 \lambda_{a}(\varphi)} *\left[Q_{s} f_{s}^{-2} d u \wedge \tau\left(I_{s}\right)\right]=\bar{Q}_{s} \tau\left(\bar{I}_{s}\right),
$$

$s \in S_{m}$, where $\bar{Q}_{s}=Q_{s} \varepsilon\left(I_{s}\right) \mu\left(I_{s}\right) w$ and $\mu(I)= \pm 1$ is defined by the relation $\mu(I) d u \wedge$ $\tau\left(I_{0}\right)=\tau(\bar{I}) \wedge d u \wedge \tau(I)$. The relation (5.54) may be readily obtained using the formula (5.28) from [16] (for $\gamma=\gamma_{0}$ ).

Relations for the metric follows from (5.42), (5.43) and (5.49)

$$
\begin{gathered}
g=\left(\prod_{s \in S_{*}}\left[f_{s}^{2}\left(u-u_{s}\right)\right]^{\eta_{s} d\left(I_{s}\right) \nu_{s}^{2} /(D-2)}\right)\left\{\left[f_{0}^{2}\left(u-u_{0}\right)\right]^{d_{0} /\left(1-d_{0}\right)} \mathrm{e}^{2 c^{0} u+2 \bar{c}^{0}}\right. \\
\left.\left.\times\left[w d u \otimes d u+f_{0}^{2}\left(u-u_{0}\right) g^{0}\right]+\sum_{\substack { i \neq 0 \\
\begin{subarray}{c}{s \in S_{*} \\
I_{s} \ni i{ i \neq 0 \\
\begin{subarray} { c } { s \in S _ { * } \\
I _ { s } \ni i } }\end{subarray}}\left[f_{s}^{2}\left(u-u_{s}\right)\right]^{-\eta_{s} \nu_{s}^{2}}\right) \mathrm{e}^{2 c^{i} u+2 \bar{c}^{i}} g^{i}\right\} .
\end{gathered}
$$

Thus we obtained exact solutions for multidimensional cosmology, describing the evolution of $(n+1)$ spaces $\left(M_{0}, g_{0}\right), \ldots,\left(M_{n}, g_{n}\right)$, where $\left(M_{0}, g_{0}\right)$ is an Einstein space of non-zero curvature, and $\left(M_{i}, g^{i}\right)$ are "internal" Ricci-flat spaces, $i=1, \ldots, n$; in the presence of several scalar fields and forms. The solution is presented by relations (5.44), (5.53)-(5.55) with the functions $f_{0}, f_{s}$ defined in (5.33)-(5.40) and the relations on the parameters of solutions $c^{A}, \bar{c}^{A}(A=i, \alpha), C_{0}, C_{s}\left(s \in S_{*}\right), \nu_{s}$, imposed in (5.45)-(5.48), (5.51), (5.16), respectively.

This solution describes a set of charged (by forms) overlapping $p$-branes $\left(p_{s}=d\left(I_{s}\right)-1\right.$, $s \in S_{*}$ ) "living" on submanifolds $M_{I_{s}}$ (2.19), where the sets $I_{s}$ do not contain 0, i.e. all $p$-branes live in "internal" Ricci-flat spaces.

The solution is valid if the dimensions of $p$-branes and dilatonic coupling vector satisfy the relations (5.4). In non-composite case these solutions were considered recently in [35, 36] (electric case) and [37] (electro-magnetic case). For $n=1$ see also [8, 10]. 


\subsection{Spherically symmetric solutions}

To illustrate the general solution from Subsect. 5.2 let us consider also the spherically symmetric case

$$
w=1, \quad M_{0}=S^{d_{0}} \quad g^{0}=d \Omega_{d_{0}}^{2},
$$

where $d \Omega_{d_{0}}^{2}$ is canonical metric on unit sphere $S^{d_{0}}$. We also assume that $M_{1}=\mathbf{R}$, $g^{1}=-d t \otimes d t$ and

$$
1 \in I_{s}, \quad \forall s \in S_{*},
$$

i. e. all p-branes have common time direction $t$. Let

$$
\eta_{s} \varepsilon_{s}=-1
$$

$s \in S_{*}$. For integration constants we put $\bar{c}^{A}=0$,

$$
\begin{aligned}
& c^{A}=\bar{\mu} \sum_{r \in \bar{S}} \frac{U^{r A}}{\left(U^{r}, U^{r}\right)}-\bar{\mu} \delta_{1}^{A}, \\
& C_{0}=C_{s}=\bar{\mu}^{2},
\end{aligned}
$$

where $\bar{\mu}>0$ and $\bar{S}=\{0\} \cup S$. Here $A=\left(i_{A}, \alpha_{A}\right)$ and $A=1$ means $i_{A}=1$. It may be verified that the restrictions (5.45)-(5.48) and (5.51) are satisfied identically.

We also introduce new radial variable $R=R(u)$ by relations

$$
\exp (-2 \bar{\mu} u)=1-\frac{2 \mu}{R^{\bar{d}}}, \quad \mu=\bar{\mu} \bar{d}>0, \quad \bar{d}=d_{0}-1,
$$

and put $u_{0}=0, u_{s}<0$,

$$
\frac{\left|Q_{s}\right|}{\bar{\mu} \nu_{s}} \sinh \beta_{s}=1, \quad \beta_{s} \equiv \bar{\mu}\left|u_{s}\right|, \quad s \in S_{*}
$$

Then, solutions for the metric and scalar fields (see (5.44), (5.55)) are the following

$$
\begin{array}{r}
g=\left(\prod_{s \in S_{*}} H_{s}^{2 \eta_{s} d\left(I_{s}\right) \nu_{s}^{2} /(D-2)}\right)\left\{\frac{d R \otimes d R}{1-2 \mu / R^{\bar{d}}}+R^{2} d \Omega_{d_{0}}^{2}\right. \\
\left.\left.-\left(\prod_{s \in S_{*}} H_{s}^{-2 \eta_{s} \nu_{s}^{2}}\right)\left(1-\frac{2 \mu}{R^{\bar{d}}}\right) d t \otimes d t+\sum_{\substack { i=2 \\
\begin{subarray}{c}{s \in S_{*} \\
I_{s} \ni i{ i = 2 \\
\begin{subarray} { c } { s \in S _ { * } \\
I _ { s } \ni i } }\end{subarray}} H_{s}^{-2 \eta_{s} \nu_{s}^{2}}\right) g^{i}\right\}, \\
\varphi^{\alpha}=\sum_{s \in S_{*}} \eta_{s} \nu_{s}^{2} \chi_{s} \lambda_{a_{s}}^{\alpha} \ln H_{s},
\end{array}
$$

where

$$
H_{s}=1+\frac{\mathcal{P}_{s}}{R^{\bar{d}}}, \quad \mathcal{P}_{s} \equiv \frac{\left|Q_{s}\right| \bar{d}}{\nu_{s}} e^{-\beta_{s}},
$$

$s \in S_{*}$. 
The fields of forms are given by (2.22), (2.23) with

$$
\begin{aligned}
\Phi^{s} & =\frac{\nu_{s}}{H_{s}^{\prime}} \\
H_{s}^{\prime} & =\left(1-\frac{\mathcal{P}_{s}^{\prime}}{H_{s} R^{\bar{d}}}\right)^{-1}=1+\frac{\mathcal{P}_{s}^{\prime}}{R^{\bar{d}}+\mathcal{P}_{s}-\mathcal{P}_{s}^{\prime}}, \\
\mathcal{P}_{s}^{\prime} & \equiv-\frac{Q_{s} \bar{d}}{\nu_{s}} .
\end{aligned}
$$

$s \in S_{*}$. It follows from (5.62), (5.65) and (5.68) that

$$
\left|\mathcal{P}_{s}^{\prime}\right|=\frac{\mu}{\sinh \beta_{s}}=\mathcal{P}_{s} e^{\beta_{s}}=\sqrt{\mathcal{P}_{s}\left(\mathcal{P}_{s}+2 \mu\right)},
$$

$s \in S_{*}$.

The solutions obtained describe non-extremal charged intersecting $p$-branes and agree with those ifrom Refs. [9], [14], [15] $\left(d_{1}=\ldots=d_{n}=1, \eta_{s}=+1\right)$ and [37] $\left(\eta_{s}=+1\right.$, non-composite case).

We note that Hawking temperature corresponding to the solution is (see also [15, 37])

$$
T_{H}=\frac{\bar{d}}{4 \pi(2 \mu)^{1 / \bar{d}}} \prod_{s \in S_{*}}\left(\frac{2 \mu}{2 \mu+\mathcal{P}_{s}}\right)^{\eta_{s} \nu_{s}^{2}} .
$$

Recall that $\eta_{s} \nu_{s}^{2}=\left(U^{s}, U^{s}\right)^{-1}$.

\subsection{WDW equation with fixed charges}

We may consider also another scheme based on zero-energy constraint relation (5.31). The corresponding WDW equation in the harmonic gauge reads

$$
\hat{H}_{Q} \Psi \equiv\left(-\frac{1}{2 \mu} \bar{G}^{A B} \frac{\partial}{\partial x^{A}} \frac{\partial}{\partial x^{B}}+\mu V_{Q}\right) \Psi=0,
$$

where potential $V_{Q}$ is defined in (5.30). This equation describes quantum cosmology with classical fields of forms and quantum scale factors and dilatonic fields. Such approach is equivalent to the scheme of quantization of multidimensional perfect fluid considered in 24].

Eq. (5.71) is readily solved in the orthogonal case (5.4). The basis of solutions is given by the following replacements in (5.19), (5.22), (5.24) and (5.25) respectively

$$
\begin{array}{r}
P_{s} \mapsto Q_{s}, \\
2 a R[\mathcal{G}] \mapsto 0, \\
\Psi_{s}\left(z^{s}\right) \mapsto B_{\omega_{s}}^{s}\left(\sqrt{\eta_{s} \varepsilon_{s} Q_{s}^{2}} \frac{\mathrm{e}^{q_{s} z^{s}}}{q_{s}}\right), \\
\omega_{s} \mapsto \sqrt{-2 \eta_{s} \mathcal{E}_{s} \nu_{s}^{2}} .
\end{array}
$$

We note that recently such solution for the special case with one internal space $(n=1)$ and non-composite $p$-branes was considered in [34]. 


\section{Intersection rules for integrable configurations with orthogonal and non-orthogonal $U^{s}$}

In this section we analyze in details the orthogonality relation (5.4). According to (2.28), (2.29), (5.3) and (5.4) there exist some obstacles for the existence of binary configurations, i. e. solutions with two $p$-branes. For these reason some restrictions on the model (2.1) (e. g. on dimension $D$, ranks of forms $n_{a}$ and scalar products for dilatonic couplings $\lambda_{a} \cdot \lambda_{b}$ ) and the manifold (2.10) (e.g. on dimensions $d_{0}, d_{1}, \ldots d_{n}$ ) should be imposed. For example, there are no binary configurations when: i) all $\lambda_{a} \cdot \lambda_{b}$ are irrational; ii) $d_{0}$ is big enough, i. e. the dimension of "internal" space (where all $p$-branes live) $D-d_{0}-1$ is too small; iii) dimensions of factor spaces $d_{i}(i \geq 1)$ do not fit the ranks of forms $n_{a}$ (see $(2.28)$ and $(2.29)$ ).

In subsection 6.1 we propose a sufficiency condition for the existence of "full" spectrum of $p$-brane pairs (see Propositions 1,2). This condition is formulated in terms of so-called fundamental matrix of the model.

In subsection 6.2 we suggest a chain of $D \geq 11$ models ("beautiful" models). These models satisfy conditions of Proposition 1 for $d_{0}=2$. They may be considered as a nice polygon for investigating of solutions with intersecting $p$-branes and possible generalization to higher dimensional supergravitational and super- $p$-brane models $\left(F_{D^{-}}\right.$or $M_{D^{-}}$theories).

In subsection 6.3 we consider the generalization of the orthogonality relation leading to some other integrable Euclidean Toda-like Lagrangians and find corresponding intersection rules. This result may be considered as a "bridge" between Toda lattices (open, closed etc.) and intersecting $p$-branes. It opens a new area for using of Lie algebras (e. g. affine Lie algebras etc.) in the (classical and quantum) models with $p$-branes.

\subsection{Orthogonal $U^{s}$}

In Sect. 5 we obtained exact cosmological solutions for the model (2.1) with $\Lambda=0$ defined on the manifold (2.10). In (2.10) $M_{0}$ is an Einstein space of non-zero curvature and hence $d_{0}=\operatorname{dim} M_{0}>1$. All $p$-branes "live" in internal space

$$
\bar{M}=M_{1} \times \ldots \times M_{n}
$$

$\left(\left(M_{i}, g^{i}\right)\right.$ are Ricci-flat). Here we treat the existence of the solutions with two $p$-branes. Let us consider such solution with set of indices $S_{*}=\left\{s_{1}, s_{2}\right\}, s_{1} \neq s_{2}$, where $s_{1}=$ $\left(a_{s_{1}}, v_{s_{1}}, I_{s_{1}}\right), s_{1}=\left(a_{s_{1}}, v_{s_{1}}, I_{s_{1}}\right) ; a_{s_{1}}, a_{s_{2}} \in \Delta ; v_{s_{1}}, v_{s_{2}} \in\{e, m\} ; I_{s_{1}}, I_{s_{2}} \subset\{1, \ldots, n\}$.

The orthogonality relation (5.4) defines the intersections rule

$$
d\left(I_{s_{1}} \cap I_{s_{2}}\right)=\Delta\left(s_{1}, s_{2}\right),
$$

where

$$
\Delta\left(s_{1}, s_{2}\right) \equiv \frac{d\left(I_{s_{1}}\right) d\left(I_{s_{2}}\right)}{D-2}-\chi_{s_{1}} \chi_{s_{2}} \lambda_{a_{s_{1}}} \cdot \lambda_{a_{s_{2}}},
$$

$s_{1} \neq s_{2}$. It follows from $(6.2)$ that in orthogonal case

$$
\Delta\left(s_{1}, s_{2}\right) \in \mathbf{Z}_{+} \equiv\{0,1,2, \ldots\} .
$$


The intersection symbol (6.3) is symmetric: $\Delta\left(s_{1}, s_{2}\right)=\Delta\left(s_{2}, s_{1}\right)$. In orthogonal case it satisfies the obvious restrictions

$$
\begin{array}{r}
\Delta\left(s_{1}, s_{2}\right) \leq \min \left(d\left(I_{s_{1}}\right), d\left(I_{s_{2}}\right)\right), \\
d\left(I_{s_{1}} \cup I_{s_{2}}\right)=d\left(I_{s_{1}}\right)+d\left(I_{s_{2}}\right)-\Delta\left(s_{1}, s_{2}\right) \leq D-d_{0}-1=\operatorname{dim} \bar{M} .
\end{array}
$$

The latter follows from the inclusion $M_{I_{s_{1}} \cup I_{s_{2}}} \subset \bar{M}$.

We recall that dimensions $d\left(I_{s}\right)$ obey the relations (2.28) and (2.29) that may be written as follows

$$
d\left(I_{s}\right)=\bar{D} \bar{\chi}_{s}+\bar{n}_{a_{s}} \chi_{s}
$$

where $\bar{D}=D-2, \bar{n}_{a}=n_{a}-1$ and $\bar{\chi}_{s}=\frac{1}{2}\left(1-\chi_{s}\right)=0,1\left(\chi_{s}=1,-1\right)$ for $v_{s}=e, m$ respectively.

Let

$$
N(a, b) \equiv \frac{\left(n_{a}-1\right)\left(n_{b}-1\right)}{D-2}-\lambda_{a} \cdot \lambda_{b}
$$

$a, b \in \Delta$. We call $(N(a, b))$ fundamental matrix of the model (2.1).

¿From (6.3) and (6.7) we obtain

$$
\Delta\left(s_{1}, s_{2}\right)=\bar{D} \bar{\chi}_{s_{1}} \bar{\chi}_{s_{2}}+\bar{n}_{a_{s_{1}}} \chi_{s_{1}} \bar{\chi}_{s_{2}}+\bar{n}_{a_{s_{2}}} \chi_{s_{2}} \bar{\chi}_{s_{1}}+N\left(a_{s_{1}}, a_{s_{2}}\right) \chi_{s_{1}} \chi_{s_{2}}
$$

or, more explicitly,

$$
\begin{aligned}
\Delta\left(s_{1}, s_{2}\right)=\quad & N\left(a_{s_{1}}, a_{s_{2}}\right), \quad v_{s_{1}}=v_{s_{2}}=e ; \\
& \bar{n}_{a_{s_{1}}}-N\left(a_{s_{1}}, a_{s_{2}}\right), \quad v_{s_{1}}=e, v_{s_{2}}=m ; \\
& \bar{D}-\bar{n}_{a_{s_{1}}}-\bar{n}_{a_{s_{2}}}+N\left(a_{s_{1}}, a_{s_{2}}\right), \quad v_{s_{1}}=v_{s_{2}}=m .
\end{aligned}
$$

Thus $N(a, b)$ is the dimension of intersection for two electric p-branes charged by forms $F^{a}$ and $F^{b}$ respectively. Fundamental matrix defines the intersection rules in electromagnetic case too.

Remark 1. The matrix (6.8) is unchanged if $\bar{n}_{a} \mapsto k \bar{n}_{a} \bar{D} \mapsto k^{2} \bar{D}, k \in \mathbf{N}$. For example, we may consider the chain of models with $D=9 k^{2}+2, n=\operatorname{rank} F=3 k+1$ originating ifrom truncated bosonic part of $D=11$ supergravity.

Proposition 1. Let matrix (6.8) satisfies the following restrictions

$$
\begin{aligned}
& \text { A) } \quad N\left(a_{1}, a_{2}\right) \in \mathbf{Z}_{+} ; N\left(a_{1}, a_{2}\right) \geq d_{0}-1 ; \\
& \text { (B) } \quad N\left(a_{1}, a_{2}\right) \leq \min \left(n_{a_{1}}, n_{a_{2}}\right)-d_{0} ; \\
& \text { (C) } \quad N\left(a_{1}, a_{2}\right) \geq n_{a_{1}}+n_{a_{2}}-D+d_{0}-1
\end{aligned}
$$

for any $a, b \in \Delta\left(d_{0}>1\right)$. Then $\Delta\left(s_{1}, s_{2}\right)$ ¿from (6.9) satisfies consistency relations (6.4)- (6.6) for any $s_{1} \neq s_{2}$ (with $d\left(I_{s}\right)$ from (6.7)).

Proof. Let us consider three cases: (ee) $v_{s_{1}}=v_{s_{2}}=e ;(\mathrm{em}) v_{s_{1}}=e, v_{s_{2}}=m ;(\mathrm{mm})$ $v_{s_{1}}=v_{s_{2}}=m$. Relation (6.4) follows ifrom restrictions (A), (B) and (C) for (ee), (em) and $(\mathrm{mm})$ cases respectively. Analogously (6.5) follows ifrom $(\mathbf{B}),(\mathbf{C})$ and $(\mathbf{B})$ and (6.6) follows ifrom (C), (B) and (A) for (ee), (em) and (mm) cases respectively. Proposition is proved. 
¿From relation $(\mathbf{B})$ and $d_{0}>1$ we get

$$
K(a) \equiv n_{a}-1-N(a, a)>0
$$

and hence

$$
\left(U^{s}, U^{s}\right)=K\left(a_{s}\right)>0 .
$$

Thus, inequalities (5.5) are satisfied.

Definition 1. The model (2.1) with $\Lambda=0$ defined on the manifold (2.10) is called binary complete if for any $a_{1}, a_{2} \in \Delta, v_{1}, v_{2} \in\{e, m\}$ there exists cosmological solution from 5.2.1 with two intersecting p-branes defined by indices $s_{1}=\left(a_{1}, v_{1}, I_{1}\right) \neq s_{2}=$ $\left(a_{2}, v_{2}, I_{2}\right)$.

For binary complete model we have the maximal number $|\Delta|(2|\Delta|+1)$ of different types of binary configurations (formed by $|\Delta|$ electric and $|\Delta|$ magnetic $p$-branes).

Definition 2. The matrix (6.8) satisfying the restrictions (A), (B) and (C) of Proposition 1 is called $d_{0}$-proper.

¿From Proposition 1 and (6.14) we obtain

Proposition 2. The model (2.1) with $\Lambda=0$ defined on the manifold (2.10) with $M_{1}=\ldots M_{n}=\mathbf{R}\left(n=D-1-d_{0}, d_{0}>1\right)$ is binary complete if fundamental matrix (6.8) of this model is $d_{0}$-proper.

In the next subsection we apply these propositions for $D=11$ supergravity and the chain of $D>11$ models.

\subsection{Example: chain of $B_{D}$-models}

Let us consider the action in dimension $D$

$$
S_{D}=\int_{M} d^{D} z \sqrt{|g|}\left\{R[g]+g^{M N} \partial_{M} \vec{\varphi} \partial_{N} \vec{\varphi}-\sum_{a=4}^{D-7} \frac{1}{a !} \exp \left[2 \vec{\lambda}_{a} \vec{\varphi}\right]\left(F^{a}\right)^{2}\right\},
$$

where $\vec{\varphi}=\left(\varphi^{1}, \ldots, \varphi^{l}\right) \in \mathbf{R}^{l}, \vec{\lambda}_{a}=\left(\lambda_{a 1}, \ldots, \lambda_{a l}\right) \in \mathbf{R}^{l}, l=D-11, \operatorname{rank} F^{a}=a$, $a=4, \ldots, D-7$. Here vectors $\vec{\lambda}_{a}$ satisfy the relations

$$
\begin{aligned}
& \vec{\lambda}_{a} \vec{\lambda}_{b}=N(a, b)-\frac{(a-1)(b-1)}{D-2}, \\
& N(a, b)=\min (a, b)-3,
\end{aligned}
$$

$a, b=4, \ldots, D-7$.

The vectors $\vec{\lambda}_{a}$ are linearly dependent

$$
\vec{\lambda}_{D-7}=-2 \vec{\lambda}_{4}
$$

For $D>11$ vectors $\vec{\lambda}_{4}, \ldots, \vec{\lambda}_{D-8}$ are linearly independent.

The model (6.15) contains $l$ scalar fields with negative kinetic term (i.e. $h_{\alpha \beta}=-\delta_{\alpha \beta}$ in (2.1) coupled with $l+1$ forms. For $D=11(l=0)$ the model (6.15) coincides with truncated bosonic sector of $D=11$ supergravity ("truncated" means without ChernSimons term). For $D=12(l=1)$ (6.15) coincides with truncated $D=12$ model from 29. (see also [16]). We call the model (6.15) $B_{D}$-model. 
The matrix (6.17) is fundamental matrix (see (6.8)) of the model (since $\lambda_{a} \cdot \lambda_{b}=-\overrightarrow{\lambda_{a}} \overrightarrow{\lambda_{b}}$, $\left.n_{a}=a\right)$.

The dimensions of $p$-brane worldsheets are (see (6.7))

$$
\begin{aligned}
& d(I)=\quad 3, \ldots, D-8, \quad I \in \Omega_{a, e} \\
& D-5, \ldots, 6, \quad I \in \Omega_{a, m}
\end{aligned}
$$

We have $(l+1)$ electric and $(l+1)$ magnetic $p$-branes $p=d(I)-1$. The intersection rules are given by (6.9). For $D=12$ see also [16].

Fundamental matrix (6.17) is $d_{0}$-proper, iff $d_{0}=2$ and hence $B_{D}$-model is binary complete if the manifold decomposition (2.10) with $M_{1}=\ldots M_{n}=\mathbf{R}, n=D-3, d_{0}=2$ is chosen. So, for spherically-symmetric solutions the binary-completeness takes place only in "non-Tangerlini" case $M_{0}=S^{2}$.

We note that in $B_{D^{-} \text {-models }}\left(U^{s}, U^{s}\right)=2>0$, since

$$
K(a)=2
$$

(see (6.14)) for all $a=4, \ldots, D-7$.

Remark 2. Here two problems arise. The first one is the existence of the chain of extensions

$$
B_{D} \longleftarrow \hat{B}_{D} \longleftarrow S G_{D} \longleftarrow M_{D}=F_{D}
$$

where $M_{D}=F_{D}$ is $M$ - or $F$-theory in dimension $D, S G_{D}$ is supergravitational (field) theory coinciding with low-energy limit of $F_{D}$ and $\hat{B}_{D}$ is the bosonic sector of $S G_{D}$. $\hat{B}_{D^{-}}$ action is the sum of $B_{D}$-action and Chern-Simons terms. The second problem is the existence of relations between $B_{D}$ models via dimensional reduction

$$
B_{11} \longleftarrow B_{12} \longleftarrow B_{13} \longleftarrow B_{14} \longleftarrow \ldots
$$

For $B_{11} \longleftarrow B_{12}$ see [29].

We note that $B_{D}$ models have also rather interesting classes of Toda lattice solutions. The intersection rules for these "Toda p-branes" may be obtained using general relations described in the next subsection. (To our knowledge $p$-brane solutions governed by open Toda lattices with $a_{n}$ Lie algebras were studied first in [8, 10].)

\subsection{Generalizations to Toda lattices}

Let us consider the Lagrange system (5.29), (5.30), where $V$ (curvature part of potential) is defined in (5.6). Here we put

$$
\left(U^{s}, U^{s}\right)>0, \quad s \in S_{*} .
$$

The Lagrangian (5.29) may be "block-diagonalized" in $z$-variables (5.11) satisfying (5.12), (5.13), $\eta_{s}=+1$ and

$$
B_{s^{\prime}}^{s} z^{s^{\prime}}=U^{s}(x)
$$

$s \in S_{*}$, instead (5.14). Vectors $\vec{B}^{s}=\left(B_{s^{\prime}}^{s}\right)$ obey the relations

$$
\vec{B}^{s_{1}} \vec{B}^{s_{2}}=\left(U^{s_{1}}, U^{s_{2}}\right),
$$


$s_{1}, s_{2} \in S_{*}$. In $z$-variables the Lagrangian (5.29) reads

$$
L_{Q}=L_{0}+L_{T, Q}+L_{f}
$$

where

$$
\begin{array}{r}
L_{0}=-\frac{1}{2}\left(\dot{z}^{0}\right)^{2}-\frac{1}{2} w \xi_{0} d_{0} \mathrm{e}^{2 q_{0} z^{0}}, \\
L_{T, Q}=\frac{1}{2} \sum_{s \in S_{*}}\left(\dot{z}^{s}\right)^{2}-\frac{1}{2} \sum_{s \in S_{*}} \varepsilon_{s} Q_{s}^{2} \exp \left(2 \vec{B}^{s} \vec{z}\right), \\
L_{f}=\frac{1}{2} \eta_{a b} \dot{z}^{a} \dot{z}^{b},
\end{array}
$$

$\vec{z}=\left(z^{s}\right) ; \varepsilon_{s} Q_{s} \neq 0, s \in S_{*}$.

Thus, the problem of integrability of the Lagrange system (6.27) is reduced to the problem of integrability of the Euclidean Toda-like system with the Lagrangian (6.29).

Let vectors $\vec{B}^{s}$ (or, equivalently, $U^{s}$ ), $s \in S_{*}$, are linearly independent. According to Adler-van-Moerbeke criterion [30] the equations of motion corresponding to (6.29) are integrable in quadratures if and only if

$$
\frac{2 \vec{B}^{s_{1}} \vec{B}^{s_{2}}}{\vec{B}^{s_{2}} \vec{B}^{s_{2}}}=\frac{2\left(U^{s_{1}}, U^{s_{2}}\right)}{\left(U^{s_{2}}, U^{s_{2}}\right)}=C_{s_{1} s_{2}}
$$

where $C=\left(C_{s_{1} s_{2}}\right)$ is the Cartan matrix for some semisimple Lie algebra $\mathbf{g}$. When $\mathbf{g}$ is simple $L_{T, Q}$ describes Toda lattice corresponding to $\mathrm{g}$ [31- 33. ¿From (5.4), (6.14), (6.3) and (6.31) we obtain the intersection rules

$$
d\left(I_{s_{1}} \cap I_{s_{2}}\right)=\Delta\left(s_{1}, s_{2}\right)+\frac{1}{2} K\left(a_{s_{2}}\right) C_{s_{1} s_{2}},
$$

$s_{1} \neq s_{2}$, where $\Delta\left(s_{1}, s_{2}\right)$ is defined in (6.3) and $K(a)$ in (6.13).

When $\operatorname{rank}\left(\vec{B}^{s}, s \in S_{*}\right)=\left|S_{*}\right|-1$ (i.e. only one vector linearly depends upon others) the Adler-van-Moerbeke criterion has the form (6.31) with the Cartan matrix corresponding to some affine Lie algebra [30]. In this case a closed Toda lattice arises.

Thus, when relations $(6.13)$ and $(6.32)$ are satisfied, the cosmological model is integrable since it is reduced effectively to (open or closed) Toda lattice.

Uninorm models. Let $K(a)=K, a \in \Delta$, and $|S| \geq 2$. Then from (6.32) we get that the Cartan matrix is symmetric. Hence $\mathbf{g}$ is of A-D-E type (or simply laced), i.e. it belongs to one of $a_{n}, d_{n}, e_{n}$ series. In this case $C_{s_{1} s_{2}}=0,-1$, for $s_{1} \neq s_{2}$ and -1 takes place for some $s_{1} \neq s_{2}$. Let $\Delta\left(s_{1}, s_{2}\right) \in \mathbf{Z}$. Then, it follows from (6.32) that $K$ should be even.

Example 1. In $D=11$ supergravity $K(a)=2$. For $S=\left\{s_{1}, s_{2}\right\}$ and $\mathbf{g}=a_{2}=\operatorname{sl}(3)$ we get the intersection rule

$$
d\left(I_{s_{1}} \cap I_{s_{2}}\right)=\Delta\left(s_{1}, s_{2}\right)-1=0,1,3
$$

for $\left\{d\left(I_{s_{1}}\right), d\left(I_{s_{2}}\right)\right\}=\{3,3\},\{3,6\},\{6,6\}$ respectively. We see that two membranes are intersecting in a point. 
Example 2. Let us consider $D=11$ supergravity and put $|S|=n=3, d_{1}=d_{2}=$ $d_{3}=3, d_{0}=1$. Let $\Omega_{e}=\{1,2,3\}$, i.e. we consider three non-intersecting electric 2-branes (Euclidean for $w=-1$ ) attached to $M_{1}, M_{2}, M_{3}$ respectively. In this case $C_{s_{1} s_{2}}=-1$ for $s_{1} \neq s_{2}$ (the Dynkin diagram is a triangle) and the Lagrangian $L_{T, Q}$ from (6.29) is closed Toda-lattice Lagrangian corresponding to affine Lie algebra $a_{2}^{(1)}$. (In this case curvature term in Lagrangian $L_{0}$ from (5.28) is absent and (5.13) should be modified.) The corresponding solutions are expressed in terms of $\theta$-functions [38].

Example 3. Let $K(a)=2$ and $n_{a}=1$ for all $a \in \Delta$. ¿From (6.3) and (6.32) we get

$$
\chi_{s_{1}} \chi_{s_{2}} \lambda_{a_{s_{1}}} \cdot \lambda_{a_{s_{2}}}=C_{s_{1} s_{2}}
$$

This relation implies the appearance of $E_{N}$ (open) Toda lattices in maximal supergrativies in $D$ dimensions coming from $D=11$ supergravity [34].

We see, that the appearance of A-D-E algebras seems to be rather typical for supergravitational models (with $K(a)=2$ ).

\section{Conclusions}

In this paper we obtained exact solutions to Einstein and Wheeler-De Witt equations for the multidimensional cosmological model describing the evolution of $n$ Ricci-flat spaces and one Einstein space $M_{0}$ of non-zero curvature in the presence of composite electromagnetic $p$-branes. The solutions were obtained in orthogonal case (5.4), when $p$-branes do not "live" in $M_{0}$. As an illustration we singled out the spherically-symmetric solutions with intersecting non-extremal $p$-branes.

In classical case we reduced the model to the Euclidean Toda-like system. Applying the Adler-van-Moerbeke criterion we obtained (Toda) intersection rules (6.32) that imply the reduction of the cosmological model to (open or closed) Toda lattice. For uninorm models $(K(a)=K, a \in \Delta)$ simply laced (A-D-E) Lie algebras appear. We gave some examples of reduction to Toda lattice (e.g. closed one). Concrete exact solutions will be considered in separate publication.

Also we developed (general) formalism of (orthogonal) intersections using the notions of fundamental matrix and binary completeness of the model and gave examples of binary complete models for $d_{0}=\operatorname{dim} M_{0}=2$ ( $B_{D}$-models). We may suppose that investigations of intersecting (e.g. Toda) p-branes may be a powerful tool in constructing new higher dimensional models (that may have supersymmetric or non-local extensions).

\section{Acknowledgments}

This work was supported in part by DFG grants 436 RUS 113/7, 436 RUS 113/236/O(R) and by the Russian Ministry for Science and Technology, Russian Fund for Basic Research, project N 95-02-05785-a. 


\section{Appendix}

\subsection{Appendix 1: Solutions with Bessel functions}

Let us consider two differential operators

$$
\begin{array}{r}
2 \hat{H}_{0}=-\frac{\partial^{2}}{\partial z^{2}}+2 A \mathrm{e}^{2 q z} \\
2 \hat{H}_{1}=-\mathrm{e}^{q z} \frac{\partial}{\partial z}\left(\mathrm{e}^{-q z} \frac{\partial}{\partial z}\right)+2 A \mathrm{e}^{2 q z} .
\end{array}
$$

Equation

$$
H_{k} \Psi_{k}=\mathcal{E} \Psi_{k}
$$

has the following linearly independent solutions for $q \neq 0$

$$
\begin{aligned}
\Psi_{k}(z)=\mathrm{e}^{k q z / 2} B_{\omega_{k}(\mathcal{E})} & \left(\sqrt{2 A} \frac{\mathrm{e}^{q z}}{q}\right), \\
\omega_{k}(\mathcal{E}) & =\sqrt{\frac{k}{4}-\frac{2 \mathcal{E}}{q^{2}}},
\end{aligned}
$$

where $k=0,1$ and $B_{\omega}, B_{\omega}=I_{\omega}, K_{\omega}$ are modified Bessel function.

\subsection{Appendix 2: Classical solutions with orthogonal vectors}

Let

$$
L=\frac{1}{2}<\dot{x}, \dot{x}>-\sum_{s=1}^{m} A_{s} \exp \left[2<b_{s}, x>\right]
$$

be a Lagrangian, defined on $V \times V$, where $V$ is $n$-dimensional vector space, over $\mathbf{R}, A_{s} \neq 0$, $s=1, \ldots, m ; m \leq n$, and $\langle\cdot, \cdot\rangle$ is non-degenerate real-valued quadratic form on $V$. Let

$$
\begin{array}{r}
<b_{s}, b_{s}>\neq 0, \\
<b_{s}, b_{l}>=0, \quad s \neq l,
\end{array}
$$

$s, l=1, \ldots, m$. Denote

$$
\eta_{s}=\operatorname{sign}<b_{s}, b_{s}>
$$

Then, the Euler-Lagrange equations for the Lagrangian (8.6) have the following solutions [28]

$$
x(t)=-\sum_{s=1}^{m} \frac{b_{s}}{<b_{s}, b_{s}>} \ln \left|f_{s}\left(t-t_{0 s}\right)\right|+t \alpha+\beta,
$$

where $\alpha, \beta \in V$,

$$
<\alpha, b_{s}>=<\beta, b_{s}>=0,
$$


and

$$
\begin{aligned}
f_{s}(\tau)= & \left|\frac{A_{s}}{E_{s}}\right|^{1 / 2} \operatorname{sh}\left(\sqrt{C_{s}} \tau\right), C_{s}>0, A_{s} \eta_{s}<0 \\
& \left|\frac{A_{s}}{E_{s}}\right|^{1 / 2} \sin \left(\sqrt{\left|C_{s}\right|} \tau\right), C_{s}<0, A_{s} \eta_{s}<0 \\
& \left|\frac{A_{s}}{E_{s}}\right|^{1 / 2} \operatorname{ch}\left(\sqrt{C_{s}} \tau\right), C_{s}>0, A_{s} \eta_{s}>0 \\
& \left|2 A_{s}<b_{s}, b_{s}>\right|^{1 / 2} \tau, C_{s}=0, A_{s} \eta_{s}<0
\end{aligned}
$$

$C_{s}=2 E_{s}<b_{s}, b_{s}>, E_{s}, t_{0 s}$ are constants.

For the energy corresponding to the solution (8.10) we have

$$
E=\sum_{s=1}^{m} E_{s}+\frac{1}{2}\langle\alpha, \alpha>.
$$

For dual vectors $u^{s} \in V^{*}$ defined as $u^{s}(x)=<b_{s}, x>, \forall x \in V$, we have $<u^{s}, u^{l}>_{*}=<$ $\left.b_{s}, b_{l}\right\rangle$, where $\langle\cdot, \cdot\rangle_{*}$ is dual form on $V^{*}$. The orthogonality conditions (8.11) read

$$
u^{s}(\alpha)=u^{s}(\beta)=0
$$

$s=1, \ldots, m$.

\section{References}

[1] E. Cremmer, B.Julia, and J. Scherk, Phys. Lett. B76, 409 (1978).

[2] M.J. Duff, R.R. Khuri and J.X. Lu, Phys. Rep. 259, 213 (1995).

[3] K.S. Stelle, "Lectures on Supergravity p-branes ", hep-th/9701088.

[4] J.M. Schwarz, "Lectures on Superstring and M-theory Dualities", Preprint ICTP, hepth/9607201.

[5] M.J. Duff, "M-theory (the Theory Formerly Known as Strings)", Preprint CTP-TAMU33/96, hep-th/9608117.

[6] C. Hull and P. Townsend, "Unity of Superstring Dualities", Nucl. Phys. B 438, 109 (1995), Preprint hep-th/9410167;

P. Horava and E. Witten, Nucl. Phys. B 460, 506 (1996), Preprint hep-th/9510209; Preprint hep-th/9603142.

[7] C.M. Hull, "String dynamics at strong coupling" Nucl. Phys. B 468, 113 (1996);

C. Vafa, "Evidence for F-Theory", Preprint hep-th/9602022; Nucl. Phys. B 469, 403 (1996).

[8] H. Lü, C.N. Pope, and K.W. Xu, "Liouville and Toda solitons im M-theory", Preprint hep-th/9604058.

[9] M. Cvetic and A.A. Tseytlin, Nucl. Phys. B 478, 181 (1996); Preprint hep-th/9606033. 
[10] H. Lü, S. Mukherji, C.N. Pope and K.-W. Xu, "Cosmological Solutions in String Theories", Preprint hep-th/9610107.

[11] V.D. Ivashchuk and V.N. Melnikov, "Intersecting p-brane Solutions in Multidimensional Gravity and M-theory", Preprint hep-th/9612089; Gravitation and Cosmology 2, No 4, 297 (1996);

V.D. Ivashchuk and V.N. Melnikov, Phys. Lett. B (1996) to be published.

[12] I.Ya. Aref'eva and O.A. Rytchkov, "Incidence Matrix Description of Intersecting p-brane Solutions", Preprint SMI-25-96, hep-th/9612236.

[13] I.Ya. Aref'eva, M.G. Ivanov and O.A. Rytchkov, "Properties of Intersecting p-branes in Various Dimensions", Preprint SMI-05-97, hep-th/9702077.

[14] I.Ya. Aref'eva, M.G. Ivanov and I.V. Volovich, "Non-extremal Intersecting p-branes in Various Dimensions", Preprint SMI-06-97, hep-th/9702079.

[15] N.Ohta, "Intersection Rules for non-extreme p-branes", Preprint OU-HET 258, hepth/9702164.

[16] V.D. Ivashchuk and V.N. Melnikov, "Sigma-model for the Generalized Composite p-branes" Preprint hep-th/9705036, submitted to Class. and Quant. Grav..

[17] V.D. Ivashchuk, V.N. Melnikov and M. Rainer, "Multidimensional $\sigma$-models with Composite Electric p-branes", Preprint gr-qc/9705005.

[18] M. Rainer and A. Zhuk, Phys. Rev. D, 546186 (1996).

[19] V.D. Ivashchuk and V.N. Melnikov, "Multidimensional Gravity with Einstein Internal spaces", preprint RGS-96-003; Gravitation and Cosmology 2 No 3 (7), 177 (1996).

[20] C.W.Misner, In: "Magic without Magic: John Archibald Wheeler", ed. J.R.Klauder, Freeman, San Francisko, 1972.

[21] J.J. Halliwell, Phys. Rev. D D38, 2468 (1988).

[22] V.D. Ivashchuk, V.N. Melnikov and A.I. Zhuk, Nuovo Cimento B104, 575 (1989).

[23] G.W. Gibbons and S.W. Hawking, Phys. Rev. D 15, 2752 (1977).

[24] V.D. Ivashchuk and V.N. Melnikov, Int. J. Mod. Phys. D 3, No 4, 795 (1994).

[25] U. Bleyer, V.D. Ivashchuk, V.N. Melnikov and A.I. Zhuk, Nucl. Phys. B, (1994).

[26] V.D. Ivashchuk and V.N. Melnikov, Gravitation and Cosmology 1, No 3, 204 (1995).

[27] V.D. Ivashchuk and V.N. Melnikov, Class. and Quant. Grav. 11, 1793 (1994).

[28] V.R. Gavrilov, V.D. Ivashchuk and V.N. Melnikov, J. Math. Phys 36, 5829 (1995).

[29] N. Khvengia, Z. Khvengia and C.N. Pope, "Toward Field Theory of F-Theory" Preprint hep-th/9703012.

[30] M.Adler and P. van Moerbeke, Commun. Math. Phys. 83, 83 (1982). 
[31] O.I.Bogoyavlensky, Comm. Math. Phys. 51, 201 (1976).

[32] B.Kostant, Adv. in Math. 34, 195 (1979).

[33] M.A. Olshanetsky and A.M. Perelomov, Invent. Math., 54, 261 (1979).

[34] H. Lü, J. Maharana, S. Mukherji and C.N. Pope, "Cosmological Solutions, p-branes and the Wheeler De Witt Equation", Preprint hep-th/9707182.

[35] M.A. Grebeniuk, V.D. Ivashchuk and V.N. Melnikov, "Integrable Multidimensional Classical and Quantum Cosmology for Intersecting p-branes", Preprint gr-qc/9708031; submitted to Phys. Lett. B.

[36] K.A. Bronnikov, M.A. Grebeniuk, V.D. Ivashchuk and V.N. Melnikov, "Integrable Multidimensional Cosmology for Intersecting p-branes", Gravitation and Cosmology 3, No 2, 1 (1997),

[37] K.A. Bronnikov, V.D. Ivashchuk and V.N. Melnikov, "The Reissner-Nordström Problem for Intersecting Electric and Magnetic P-branes", in preparation.

[38] I.M. Krichever, Usp. Mat. Nauk, 33 (4), 215 (1978) [in Russian]. 